\title{
Benchmarking Raspberry Pi 2 Beowulf Cluster
}

\author{
Dimitrios Papakyriakou \\ mVAS Testing and Deployment \\ Engineer \\ COSMOTE Fixed and Mobile \\ Telecommunications S.A \\ Athens, Greece
}

\author{
Dimitra Kottou \\ Core Network Design \\ Engineer \\ COSMOTE Fixed and Mobile \\ Telecommunications S.A \\ Athens, Greece
}

\author{
loannis Kostouros \\ mVAS Testing and Deployment \\ Engineer \\ COSMOTE Fixed and Mobile \\ Telecommunications S.A \\ Athens, Greece
}

\begin{abstract}
This paper presents a performance benchmarking of a Raspberry Pi 2 Beowulf cluster. Parallel computing systems with high performance parallel processing capabilities has become a popular standard for addressing not only scientific but also commercial applications. The fact that the raspberry pi is a tiny and affordable single board computer (SBC), given the chance to almost everyone to experiment with knowledge and practices in a wide variety of projects akin to supercomputing to run parallel jobs. This research project involves the design and construction of a high performance Beowulf cluster, composed of 12 Raspberry Pi 2 model B computers with CPU 900MHz, 32-bit quad-core ARM Cortex-A7 CPU processors and RAM $1 \mathrm{GHz}$ each node. All of them are connected over an Ethernet Network $100 \mathrm{Mbps}$ in a parallel mode of operation so that to build a kind of supercomputer. In addition, with the help of the High Performance Linpack (HPL), we observe and depict the cluster performance benchmarking of our system by using mathematical applications to calculate the scalar multiplication of a matrix, extracting performance metrics such as runtime and GFLOPS.
\end{abstract}

\section{Keywords}

Raspberry Pi cluster, Cluster Computing, Message Passing Interface, High Performance Linpack (HPL), Benchmarking RPi clusters.

\section{INTRODUCTION}

We may not have been realized in our mind that our real world is massively parallel, where many complex and interrelated events are happening at the same time. Parallel computing compared to serial computing is much better suited to model, simulate and understand complex real world phenomena, such as Galaxy Formation, Planetary Movements, and Climate Changes. That is to say, when we deal with large and complex problems, it's impractical or impossible to solve them on a single computer, with given limited RAM memory, and even worst to deal with the so called "Grand Challenge Problems". In this case we definitely require Petaflops and Petabytes of computer resources to possibly reach a mathematical problem solution.

Nowadays, we all understand that Digital Globalization is the new era of global flows of data information and communication. Internet of Things (IoT) and Clouding are driving data complexity and advanced analytic techniques against large and diverse data sets from different sources. The Big Data era needs to embrace data parallel computing technics so that to increase the efficient data analysis, where data processing and data excavation are the main area in supercomputer applications today. Since supercomputers require an enormous amount of energy to operate, the alternative solution is to use an affordable single board computer (SBC), such as Raspberry Pi 2 Model B to experiment parallel computing topics.

Raspberry Pi (RPi) 2 Model B "Figure 1" is equipped with a $900 \mathrm{MHz}$ quad-core ARM Cortex-A7 CPU (BCM2836) and 1 GB of RAM (LP DDR2 SDRAM) [1]. The low cost of the Raspberry Pi was the driving force for us to investigate a viable option for building a high performance cluster computer and to study the Pi's ability to perform in a parallel clustering mode of operation.

An additional motivation for this project is to gain knowledge, experience and understanding of a fully functional clustering computer by the construction and using different benchmarking processes. Moreover, there is an Academic and Professional interest from the authors to investigate later on, how the parallel computing systems can be used for testing purposes in cloud environment.

At this stage in our current project we built a 12 node RPi's 2 cluster with message passing interface (MPI) configuration so that to measure the performance of the cluster with the Highperformance Linpack (HPL) benchmark.

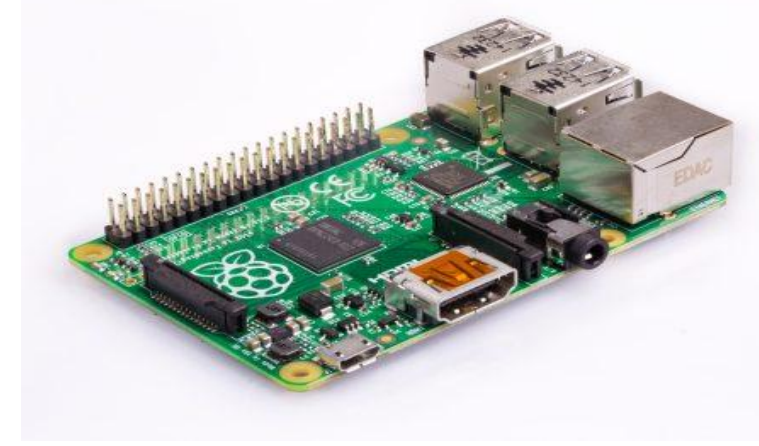

Figure 1: Single Board Computer (SBC) - Raspberry Pi 2 Model B [1].

\section{SYSTEM DESCRIPTION}

\subsection{Hardware Equipment}

The cluster is composed of 12 Raspberry Pi 2's "Figure 2", of which one RPi is the master (or head) node, responsible for distributing jobs and resources including itself in this process. The rest 11 RPi's are simply the worker nodes obeying in the master node instructions. All the nodes are stacked together in two groups of 5 RPi's each, and are connected to a 16-Port 10/100 Mbps Ethernet switch, where the maximum network throughput for any individual node is $100 \mathrm{Mbps}$. Each RPi requires a microSD card since the Pi cannot be booted without it. The size of each microSD card is $32 \mathrm{~GB}$. There is an external Hard Disk (HD) with size 320GB connected to the master node, apart from the microSD card of size $32 \mathrm{~GB}$ 
needed to boot it. Moreover, there are 3 USB cords to power the individual Pi's with 2 switch-mode power supplies of $100 \mathrm{~W}$ with $5 \mathrm{~V}$ output boosted to $5.5 \mathrm{~V}$ so as to adjust the voltage drop. In addition, there are 4 cooling FANs to provide cooling solutions to the system thermal problems and 3 voltage meters to supervise the voltage output of the switchmode power supplies.

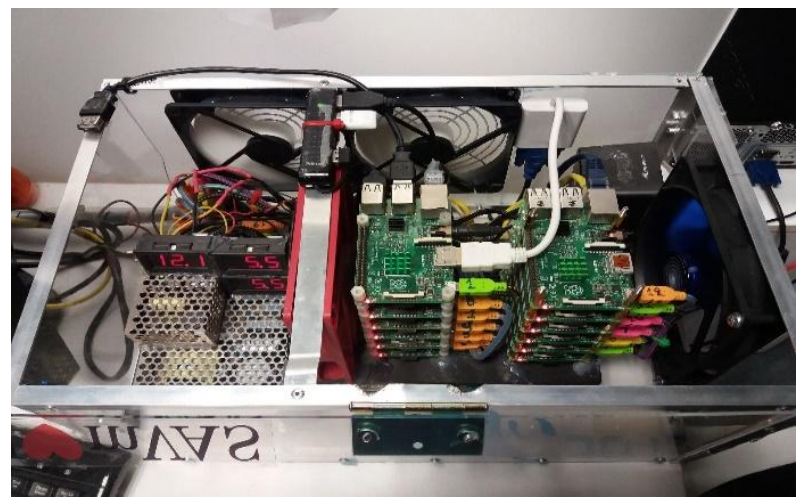

Figure 2: Construction of the Beowulf Cluster.

\subsection{Software Tools}

The Operating System used to setup the RPi's in the cluster is the Raspbian GNU/Linux 8 (Jessie) which is one of the official supported Operating System (OS) [2].

The $2^{\text {nd }}$ Software Package we needed to install is the Message Passing Interface (MPI). The MPI is not a library but a standard for development of message-passing libraries based on recommendations of the MPI Forum [3]. There are two prominent implementations of MPI that can be used on the Raspberry Pi. These are: OpenMPI and MPICH. In our case we use MPICH [4], which originally standing for Message Passing Interface Chameleon. It's an implementation of the MPI standard that supports $\mathrm{C}, \mathrm{C}++$ and FORTRAN applications. MPICH is a high performance and wide portable implementation of the Message Passing Interface (MPI) standard.

The $3^{\text {th }}$ software package we needed to install is the (GNU Compiler Collection) GCC Fortran compiler which has optimization and multi-threading features. It's the default compiler suite in High Performance Computing (HPC).

The $4^{\text {th }} \mathrm{SW}$ package we needed to install so that to configure properly the cluster is the MPI4PY which stands for MPI for Python. MPI for Python provides MPI bindings for Python. This allows any python program to use a multiple-processor configuration computer. This package is built on top of the MPI-1/2/3 specifications and provides an object-oriented interface for parallel programming in Python. It supports point-to-point (send and receive) and collective (broadcast, scatter and gather) communications for any Python object. Since Message Passing Interface (MPI) is the de facto standard for High Performance Computing (HPC) and parallel programming and the MPI4PY is well-regarded and efficient implementation of MPI for Python, we decided to use this package on the Raspberry Pi for parallel programming. The $6^{\text {th }} \mathrm{SW}$ package was the High Performance Linpack (HPL) [5]. The High Performance Linpack benchmark used to measure the performance of a HPC system. It's used by both the TOP 500 [6] and the Green 500 [7] to compile their rankings. The HPL benchmark is based on the original Linpack benchmark, measuring performance based on solving a system of linear equations using LU factorization [8].
In addition to the above software packages, we lastly needed to load the High Performance Linpack (HPL) software dependency which was the Basic Linear Algebra Subprograms (BLAS). BLAS are a set of well-defined basic linear algebra operations routines to provide standard building blocks so as to perform basic vector and matrix operations [9].

\subsection{Design and Setup}

The below mentioned "Figure 3" depicts the RPI cluster architecture diagram.

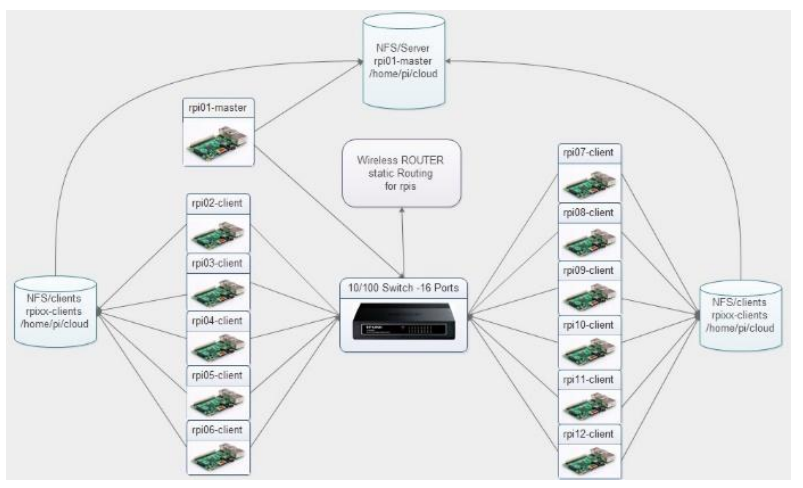

Figure 3: Beowulf cluster architecture diagram

\subsubsection{Design}

The Cluster design is composed of 12 Raspberry Pi's connected to the 16-Port 10/100 Mbps Ethernet switch. One out of the $12 \mathrm{Pi}$ is the master or head of the cluster and the rest 11 are slaves or workers. The network configuration is built with static routing namely, each node has a static IP address and the configuration is in such a way where the master can only communicate to every node with secure shell. NFS server shared folder created and configured to both master and slaves Raspberry Pi's since it's easier to setup the whole cluster from design point of view. Moreover, there is a sharing internet connection to Raspberry Pi's meaning that the slave nodes communicate with the Internet via the master node, having the ability to update the operating system with new releases and the RPi's firmware software as well. As far as the master node is concerned, the boot partition still remains in the microSD card but the root partition is located in the external Hard Disk (HD) with size 320GB. A backup and Shrink procedure to save the master node image is applied prior any significant upgrade and configuration changes so that to be able to reload the previous backup image in case of serious software crash.

\subsubsection{Setup}

The first step was to prepare the master node by loading the Raspbian GNU/Linux 8 (Jessie) operating system in the microSD card since the Pi can boot only from that card. Once we are able to boot up a single $\mathrm{Pi}$, then we install the gfortran software package and Message Passing Interface (MPI) by installing the MPICH software package and the MPI4PY software. At this point, we took the image of the microSD card and replicate it to equal microSD cards as such the number of the slave Raspberry Pi's that the cluster is composed of, so as to avoid the repetition of the work for each $\mathrm{Pi}$. The next step was to configure the network. We accessed each Pi giving a static IP address from 192.168.1.1 domain for this project, a hostname per $\mathrm{Pi}$, such as rpi01-master, rpi02client etc. The configuration of the SSH keys for each $\mathrm{Pi}$ followed, since the master node needs password less access into the slave Raspberry Pi's over Secure Shell (SSH). 
At this stage the cluster can be tested successfully provided that everything is configured correctly. Here, the Network File System (NFS) configuration took place so that to easier configure the High Performance Linpack (HPL).

The last step was to install the High Performance Linpack (HPL) with all the dependencies, such as the Basic Linear Algebra Subprograms (BLAS) development libraries in the master node. In the slave nodes, there is only a need to install the Basic Linear Algebra Subprograms (BLAS) development libraries and nothing more.

\section{PERFORMANCE EVALUATION}

The crucial aim of the running tests in the raspberry cluster is to determine if the cluster was scalable, meaning that, if the addition of more cores resulted an addition to the processing capacity of the cluster. Moreover, we had to keep in our mind that each Raspberry Pi comprises a quad-core CPU (4 threads total), meaning that 4 processes can run at the same time per RPi. As a result, 12 Pis comprises 48 cores and as a result 48 processes can be run simultaneously.

\subsection{HPL Benchmarks}

Broadly speaking, benchmarking is a process of running standard programs to evaluate the speed of a system, where in our case we use the so called High-Performance Linpack (HPL). The Linpack Benchmark is a measure of a computer's floating rate of execution and determines the upper bound of double precision floating point performance on a distributed parallel system. In other words, measures how fast a computer solves a random dense linear system of equations of order (n), $\left[A \times x=b ; \quad A \in R^{n x n} ; \quad x, b \in R^{n}\right]$ by first computing the LU factorization [10], with row partial pivoting of [ $n$ by $(n+$ 1)] coefficient matrix $[A b]=[[\mathrm{L}, \mathrm{U}] \mathrm{y}]$. Since the lower triangular factor (L) is applied to (b) as the factorization progresses, the solution $(\mathrm{x})$ is obtained by solving the upper triangular system $U \times x=y$. The lower triangular matrix (L) is left unpivoted and the array of pivots is not returned. The data is distributed onto a two-dimensional grid of processes $(P$ by $Q)$, according to the block-cyclic scheme, to ensure "good" load balance, as well as the scalability of the algorithm [11],[12]. To determine the scalability of the cluster, the problem size or matrix size $(\mathrm{N})$ was kept constant and the number of processors was gradually varied from 4 (1 $\mathrm{RPi})$ to $48(12 \mathrm{RPi})$. Since there is no formula mentioned anywhere we run the benchmark several times in order to get the best possible results of HPL benchmark. We had to fine tune several configuration parameters in the HPL.dat file, focusing especially in the Number of problems size $(\mathrm{N})$, the Number of the block size (NBs) in the grid and the Number of process grids $(P \times Q)$ [13].

Briefly, the most important parameters in HPL.dat file that we had to configure are analyzed below:

Number of problems sizes $(\mathrm{N})$. - Parameter $(\mathrm{N})$ specifies the problem size. The aim is to find the largest problem size that fits into the main memory of a specific cluster and for this reason, the main memory capacity for storing double precision (8 Bytes) numbers is calculated. That is to say, in order to get the best performance of the cluster, it's needed to have the largest problem size that can fix in the entire main RPi memory. We use an "optimal" configuration which is found by the Nelder-Mead tuning method and changes the problem size $(\mathrm{N})$ with other parameters unchanged. The max problem size is calculated as [14] suggests: $N_{\max }=$
$80 \% \sqrt{m \times n}$ where $(\mathrm{m})$ is the free memory in doubles for the machine with the least available free memory and (n) is the number of nodes. The mathematical expression can be seen as such:

$\mathrm{N}_{\max }=\sqrt{\left(\frac{\text { Memory in Gbytes } \times 1024^{3} \times \text { No of Nodes }}{8}\right)} \times \mathrm{Z} \quad$ The (N) in overall must be (80-90) \% of the size of the total memory. In particular, in our Raspberry Pi cluster, the maximum possible value for $(\mathrm{N})$ is 32105 (representing approximately the $80 \%$ of the available memory) taking into account the $12 \mathrm{RPi}$ nodes, the 4 cores per RPi node, the Memory per Node equal to $1 \mathrm{~GB}$ and the block size (NB) equal to 128 . As a rule of thumb, it's wise to use the $(\mathrm{N})$ equal to $80 \%$ of available memory in order to avoid cluster crash with errors. For a single RPi, $N_{\max } \approx 11585 \times 0.8=9268$ or alternatively we have the following,: $\mathrm{N}_{\max } \approx 11585 \times$ $0.9=10426$.

Number of block size (NBs). - The (NB) is the block size in the grid. HPL uses the block size (NB) for the data distribution and for the computational granularity. Usually block sizes giving good results are within $[96,104,112,120$, $128 \ldots 256]$ range. The principle is that smaller (NB) gives better load balance from a data distribution point of view, but it's preferred not to have very large values of (NB). From computational point of view, a too small value of (NB) will probably limit the computational performance. As a result there is a need to apply a trial and error method to find the best approach for better computational performance.

Number of process grids $(P \times Q) .-(P \times Q)$ is the size of the grid where $\mathrm{P}$ (the number of process rows) and $\mathrm{Q}$ (the number of process columns) should be close to being a "square". According to the developers of the (HPL) in [15], [16] (P) and (Q) should be approximately equal, with Q slightly larger than $\mathrm{P}$ which is equal to the number of processors that the cluster has.

Since $(\mathrm{N})$ should be $(\mathrm{NB})$ aligned [17], [18] an additional optimization is needed. For instance, if we consider $\mathrm{NB}=128$, with $12 \mathrm{RPi}$ 's, then $(\mathrm{N})$ is calculated as following:

$\mathrm{N}_{\max }=\sqrt{\left(\frac{\text { Memory in Gbytes } \times 1024^{3} \times \text { No of Nodes }}{8}\right)} \times \mathrm{Z}$

Where $(\mathrm{Z})$ is the reduction coefficient, taking values between (80-90) percent, and as a result we have below:

$\mathrm{N}=\sqrt{\left(\frac{1 \mathrm{~GB} \times 1024^{3} \times 12}{8}\right)} \times 80 \%=32105 . \quad$ Considering $\mathrm{NB}=128$, we calculate $\left(\frac{32105}{128} \times 128=250.8203125 \approx\right.$ $250)$ and next $(250 \times 128=32000)$. As a result, we optimize the parameters $\mathrm{N}=32000, \mathrm{NB}=128$ and $P \times Q=48$ in the HPL.dat when we run it for the 12 RPi's. The same optimization logic was applied when executing the benchmark with different values using all the nodes, since there is different proportion of the systems' total main memory when we use different number of RPi's in the testing.

\subsection{Results}

\subsubsection{Computing Performance vs. Number of} Node (HPL)

"Table 1" and "Table 2" depicts the detailed data of the HPL performance analysis with the parameters $(\mathrm{N}),(\mathrm{P}),(\mathrm{Q})$, and (NB) as well as the runtimes results of the cluster, required to 
solve the linear system and the achieved GFlops. In "Table 1", the HPL run with system memory utilization $(\approx 80 \%)$ compared to what "Table 2 " shows up, which is $(\approx 85 \%)$. The highest performance peaks can be seen in "Figure 4", and "Figure 5", respectively in the command line interface as well, whereas "Figure 6" and "Figure 7" depict the cluster performance results in GFlops with $80 \%$ and $85 \%$ system memory utilization. The theoretical peak performance can be determined by counting the number of floating-point additions and multiplication (in double precision), that can be completed during the cycle time of the machine. The equation (1) which gives the theoretical peak performance is depicted below:

$\mathrm{R}_{\text {peak }}[$ GFlops $]=$

Average frequency $[\mathrm{GHz}] \times$ No of Cores $\times$

operations per cycle

The efficiency of the system in percent is calculated such as $\frac{R_{\max }}{R_{\text {peak }}} \times 100$ and is dependent on the executed operations. The ARM Cortex A7 processors, used in our cluster, can process one floating-point addition in one cycle, requiring two cycles for a floating-point multiplication.

Moreover, the results in "Figure 8" show that increasing the problem size $(\mathrm{N})$, results in a better GFlops performance. At the same time we notice that increasing the problem size $(\mathrm{N})$ and the RPi's in the testing, results an increasing of the speedup of the system which can be seen easily in the "table 1 " and "table 2" as well. Apart from the cluster performance in GFlops, the investigation of the speedup when we add gradually RPi nodes in the test was a research question to be answered. The speedup $\left(S_{\mathrm{p})}\right.$ that can be achieved when the testing is run and can be defined with equation (2) as following:

$$
S_{p}=\frac{F_{p}}{F_{1}}(2)
$$

Where $\left(F_{1}\right)$ is the GFlops on a single-processor system and $\left(\mathrm{F}_{\mathrm{p}}\right)$ is the GFlops on a multiprocessor system. The theoretical maximum speedup is equal to the number of single-processor nodes. That is to say, for the two RPi nodes the value is 2 , for the four nodes the value is 4 and so on. The "Table 1 " and "Table 2" depicts the experimental results of the HPL benchmarking testing. It can be seen the increased speedup performance achieved by (HPL) testing when we gradually increase the RPi Nodes from 1 to 12 in the cluster. The outcome of the (HPL) is exactly what we were expecting since the increased RPi's means increased processors in the testing.

Table 1. Beowulf cluster setup parameters and testing results with $80 \%$ system memory utilization

\begin{tabular}{|c|c|c|c|c|c|c|}
\hline $\begin{array}{c}\text { System } \\
\text { Memory } \\
\text { utilized }\end{array}$ & $\mathbf{N}$ & $\begin{array}{c}\text { Nodes } \\
\text { used }\end{array}$ & $\mathbf{N B}$ & $\begin{array}{c}\text { Time } \\
{[\mathbf{s e c}]}\end{array}$ & GFlops & Speedup \\
\hline \multirow{5}{*}{$\approx 80 \%$} & 9216 & 1 & 128 & 587.51 & 0.888 & 1.00 \\
\cline { 2 - 7 } & 13056 & 2 & 128 & 842.38 & 1.762 & $\approx 1.98$ \\
\cline { 2 - 7 } & 18560 & 4 & 128 & 1640.98 & 2.598 & $\approx 2.92$ \\
\cline { 2 - 7 } & 22656 & 6 & 128 & 2028.69 & 3.822 & $\approx 4.30$ \\
\cline { 2 - 7 } & 26240 & 8 & 128 & 2446.26 & 4.924 & $\approx 5.54$ \\
\cline { 2 - 7 } & 29312 & 10 & 128 & 2938.29 & 5.715 & $\approx 6.43$ \\
\cline { 2 - 7 } & 32000 & 12 & 128 & 3137.71 & 6.963 & $\approx 7.84$ \\
\hline
\end{tabular}

Table 2. Beowulf cluster setup parameters and testing results with $85 \%$ system memory utilization.

\begin{tabular}{|c|c|c|c|c|c|c|}
\hline $\begin{array}{c}\text { System } \\
\text { Memory } \\
\text { utilized }\end{array}$ & $\mathbf{N}$ & $\begin{array}{c}\text { Nodes } \\
\text { used }\end{array}$ & $\mathbf{N B}$ & $\begin{array}{c}\text { Time } \\
{[\mathbf{s e c}]}\end{array}$ & GFlops & Speedup \\
\hline \multirow{5}{*}{$\approx 85 \%$} & 9984 & 1 & 128 & 702.79 & 0.944 & 1.00 \\
\cline { 2 - 7 } & 13952 & 2 & 128 & 1021.63 & 1.773 & $\approx 1.87$ \\
\cline { 2 - 7 } & 19712 & 4 & 128 & 1905.89 & 2.679 & $\approx 2.83$ \\
\cline { 2 - 7 } & 24192 & 6 & 128 & 2373.69 & 3.977 & $\approx 4.21$ \\
\cline { 2 - 7 } & 27904 & 8 & 128 & 2742.70 & 5.282 & $\approx 5.59$ \\
\cline { 2 - 7 } & 31104 & 10 & 128 & 3385.03 & 5.927 & $\approx 6.27$ \\
\cline { 2 - 7 } & 34048 & 12 & 128 & 3666.16 & 7.178 & $\approx 7.60$ \\
\hline
\end{tabular}

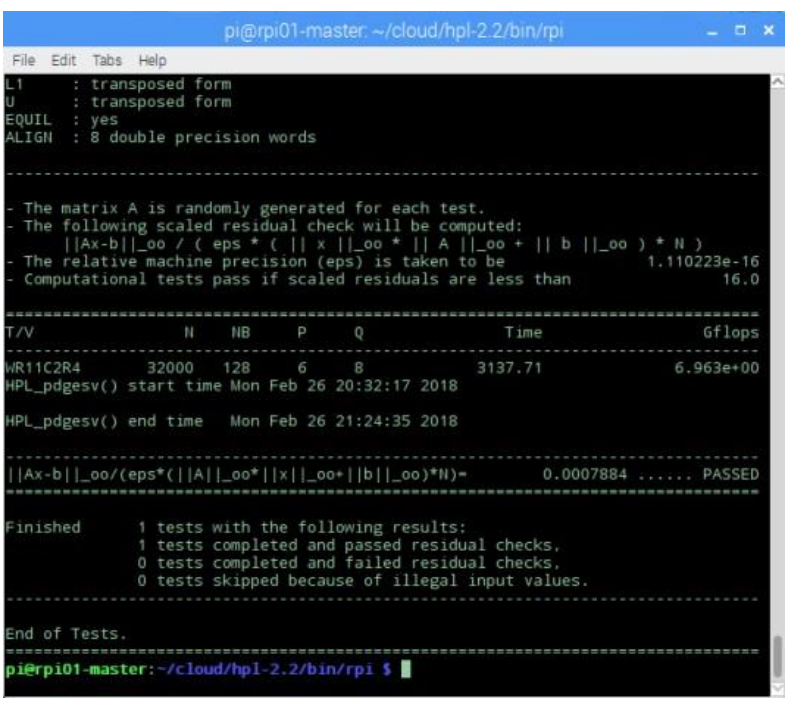

Figure 4: Highest measured cluster performance results in GFlops with $80 \%$ system memory utilization.

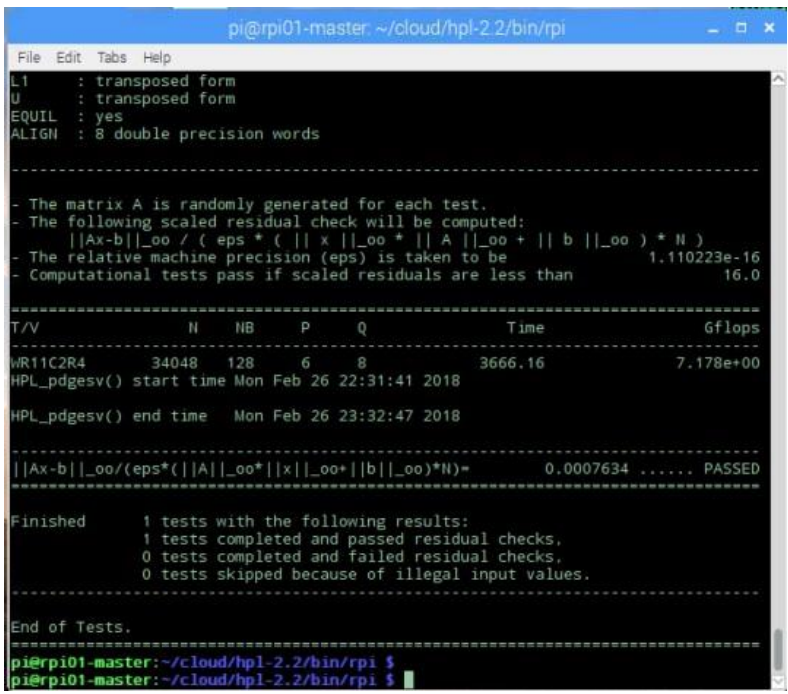

Figure 5: Highest measured cluster performance in GFlops with $85 \%$ system memory utilization. 


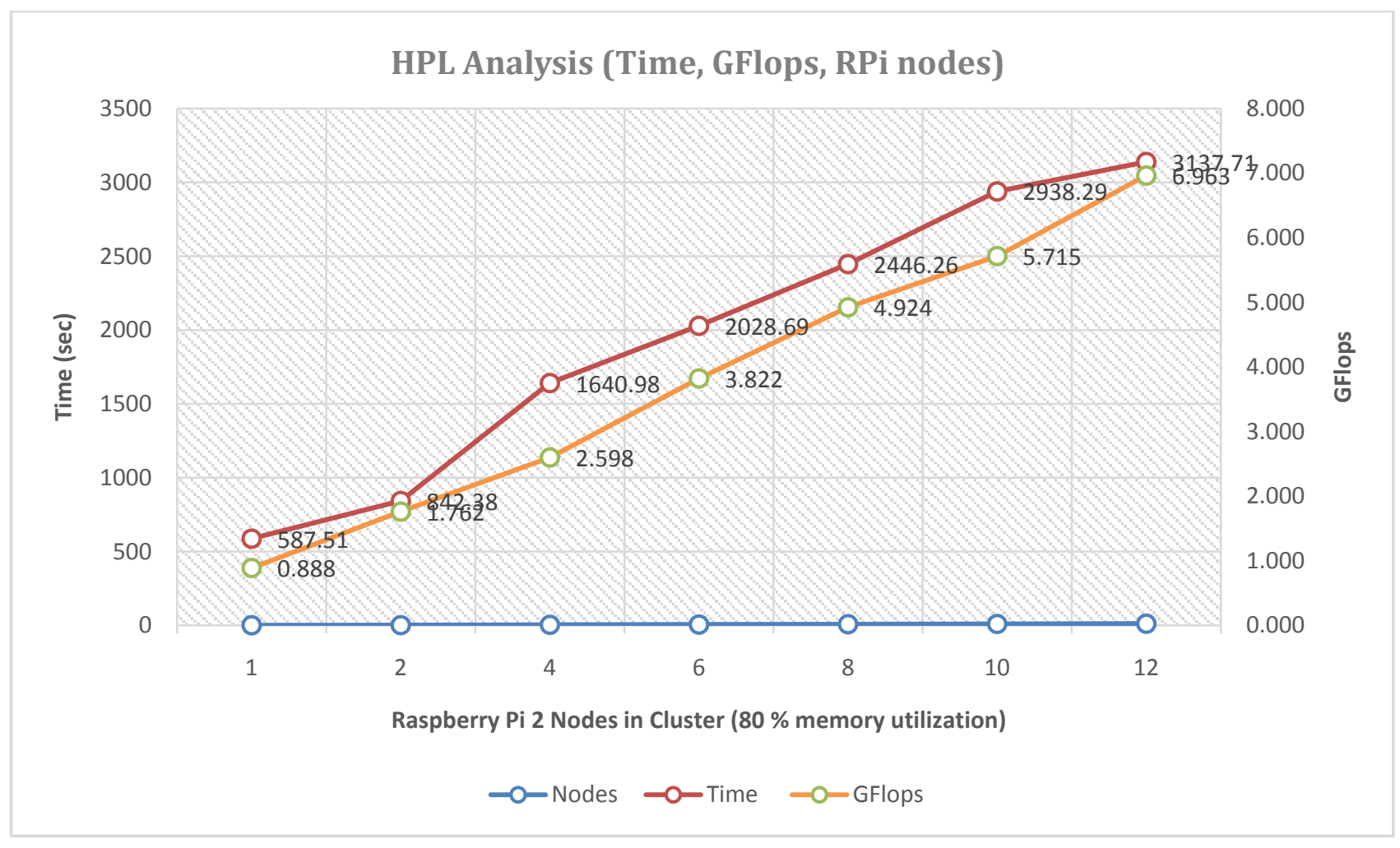

Figure 4: HPL cluster performance results in GFlops with $80 \%$ system memory utilization (4 threads per node)

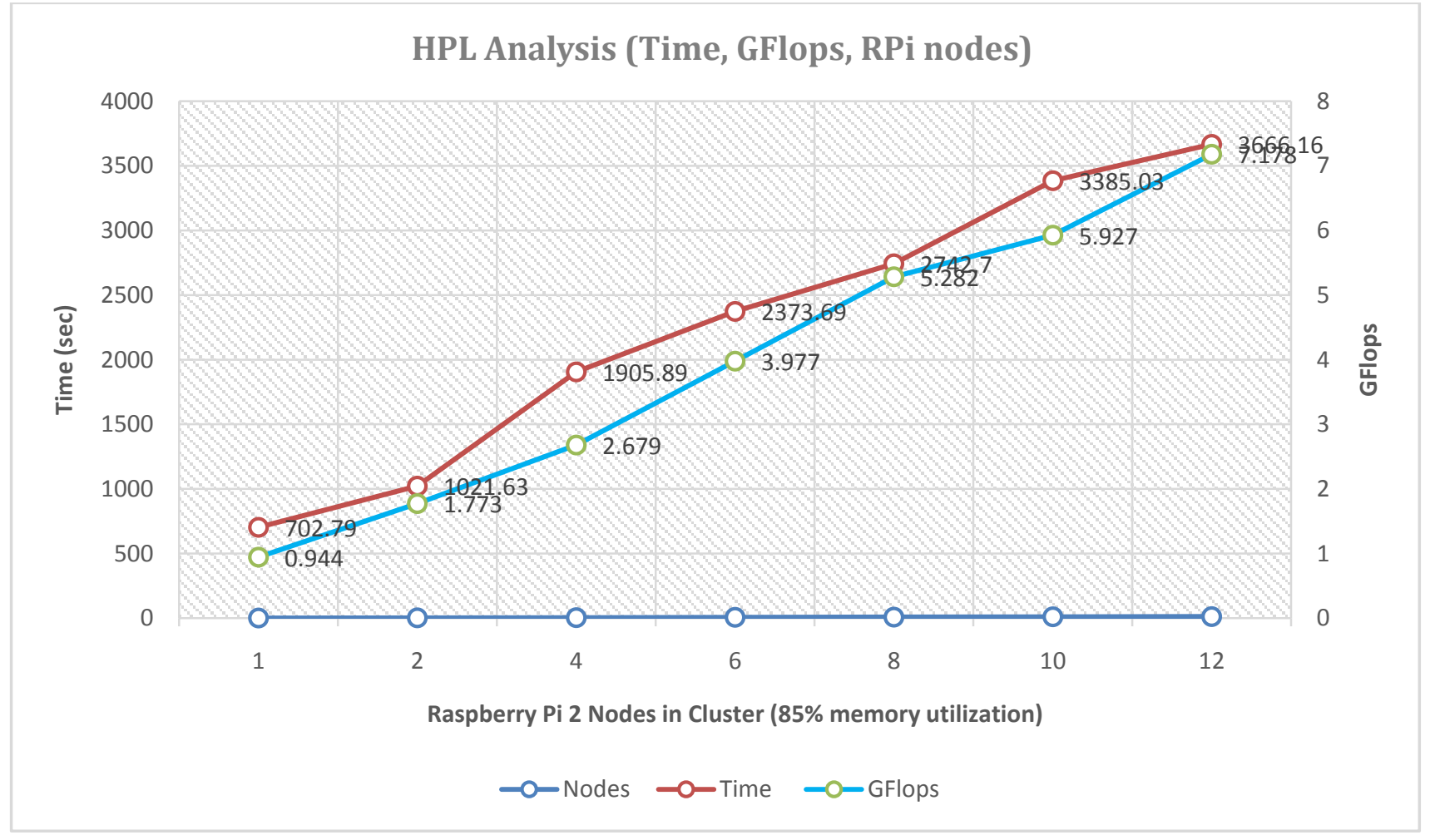

Figure 5: HPL cluster performance results in GFlops with $85 \%$ system memory utilization (4 threads per node) 


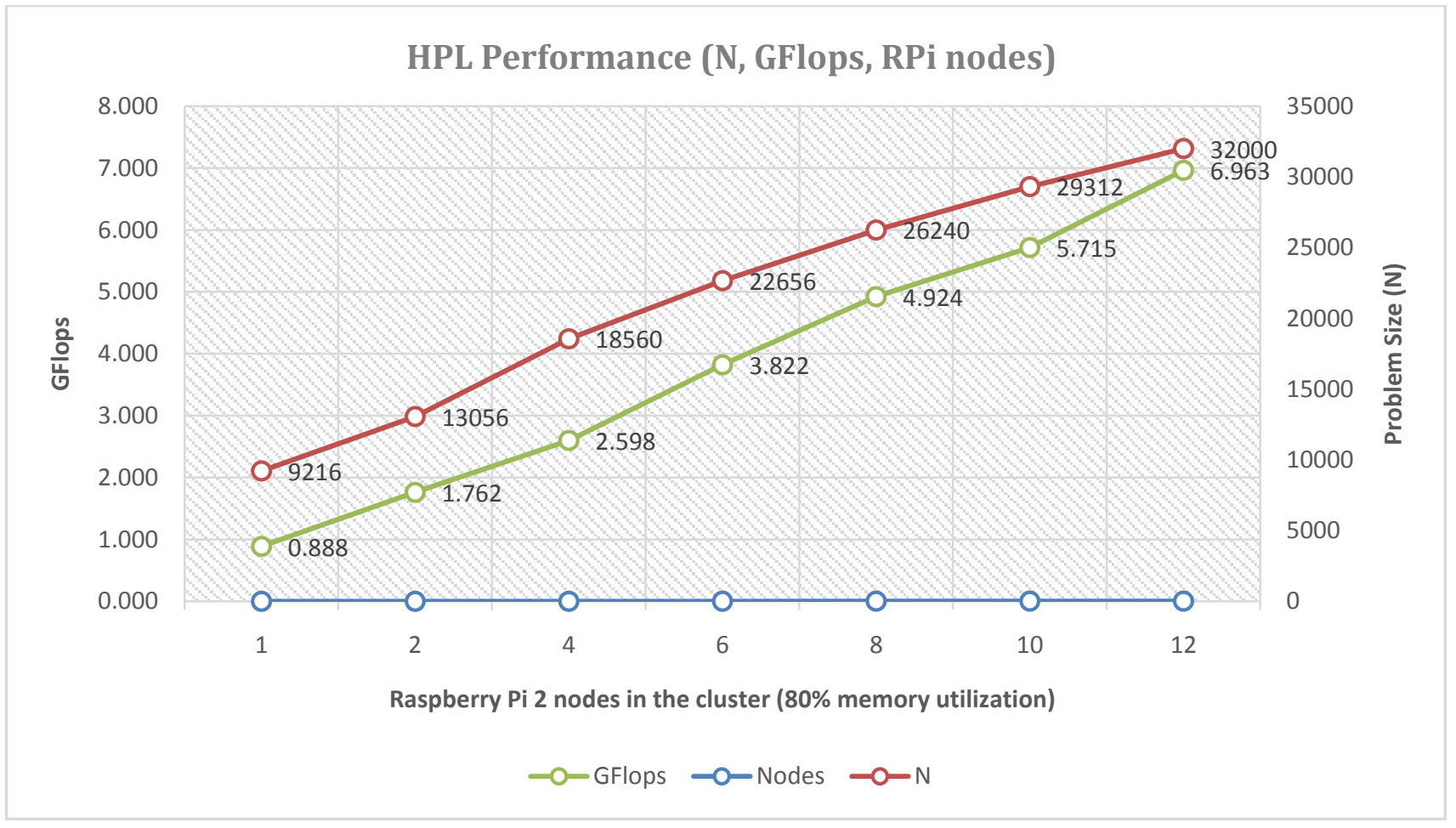

Figure 6: HPL cluster performance results compared to increased problem size (4 threads per node)

\section{CONCLUSION}

In this project, we implemented and analyzed the performance of a cluster with low cost, with embedded ARM processors and Raspberry Pi 2 platform by using the HPL benchmark. After performing this work, we can state the following observations: (a) the increase of the number of processors or the number of the RPi nodes, resulted a considerable increase in the peak performance in GFlops; (b) the increase of the RPi nodes resulted a considerable increase in speedup of the cluster; (c) In addition, increasing the problem size $(\mathrm{N})$ resulted in a better GFlops performance; (d) after the analysis and the results obtained by running the HPL benchmark, we can conclude that the ARM processors performed well when running parallel applications. Apparently, such an RPi cluster cannot be used for scientific research as a large scale supercomputer, but can be used for Academic educational reasons with no doubts. (e) We have noticed that with increasing the system memory utilization from $80 \%$ into $85 \%$, the performance in GFlops had been better -or slightly better- as expected to be, but the speedup was not. The explanation could be that the on-board (10/100 Mbps) Ethernet limits the cluster ability to outperform due to interconnection limits, meaning how quickly nodes/processes could communicate with each other. Obviously, the limitation of the 1 GB memory is an obstacle to achieve better performance but the cluster is scalable with no doubts.

Despite the limitations the RPi's depicted, since it's a single board computer (SBC) with embedded RAM, the Beowulf cluster can be used in a professional manner for automation testing purposes. In our job, we are moving from manual testing to automation testing procedures with agile methodologies. The parallel computing systems can be used as a centralized architecture to perform, in a large scale, automation testing in cloud environment with Internet of Things (IoT) applications by using for instance Python programming. This idea has been applied successfully by the authors in functional testing used in the Telecom sector and in particular in Value Added Services (VAS) Nodes. Linux and Python used with incredible performance in limited functional tests, using the parallel computing feature itself to increase the performance or in a way where the master node guides the clients to perform different set of testing batches, in different Telecom nodes at the same time.

In addition, for the authors the project had been extremely educational to see in practice how to build and analyze a parallel computing system with low cost and affordable single board computers (SBCs) and how the parallel computing systems can be used for testing purposes.

\section{FUTURE WORK}

Since our primary job is the design, testing and deployment in the Telecom Core Network and Value Added Services (VAS) domain, we are very interested in researching the Telecom cloud environment with High Performance Parallel Computing Systems. Our first attempt was to design and deploy an affordable parallel computing system using Raspberry's Pi 2 so that to move forward a few steps the idea of using cluster architectures in automation testing. The next step is to carry on with building a High Performance Hadoop cluster so that to research and analyze the cluster processing capabilities of large amount of data under the scope of Big Data Analytics. The Internet of Things (IoT) is served by cloud environments producing huge amount of data, especially in the Telecom sector where High Performance Computing Systems are needed and in turn there is a challenge opportunity to investigate not only the design and architecture but also the Automation Testing Methodologies in clouding.

\section{ACKNOWLEDGMENTS}

We express our sincere gratitude to all multi Value Added Services (mVAS) team for the precious suggestions, 
motivation and knowledge contribution for the successful completion of this project.

\section{REFERENCES}

[1] Raspberry Pi 2 Model B. [Online]. Available: https://www.raspberrypi.org/products/raspberry-pi-2model-b/

[2] Raspberry Pi 2 Model B. Operating System. [Online]. Available: https://www.raspberrypi.org/downloads/

[3] MPI. MPI Forum. [Online]. Available: http://mpiforum.org/

[4] MPI. MPICH. [Online]. Available: https://www.mpich.org/

[5] Netlib. HPL. [Online]. Available: http://www.netlib.org/benchmark/hpl/

[6] Top500.org. Top500 lists. [Online]. Available: https://www.top500.org/.

[7] Green500.org. Green500 lists. [Online]. Available: https://www.top500.org/green500/.

[8] LU factorization. [Online]. Available: https://www.geeksforgeeks.org/l-u-decompositionsystem-linear-equations/

[9] Netlib. Netlib blas, [Online]. Available: http://www.netlib.org/blas/.

[10] Mathematics. LU Decomposition of a System of Linear Equations. [Online]. Available: https://www.geeksforgeeks.org/l-u-decompositionsystem-linear-equations/
[11] Dunlop, D., Varrette, S. and Bouvry, P. 2010. Deskilling HPL, Vol. 6068 of Lecture Notes in Computer Science, Springer, Heidelberg, Berlin, 102-114.

[12] Luszczek, P., Dongarra, J., Koester, D., Rabenseifner, R., Lucas, B., Kepner, J., McCalpin, J., Bailey, D. and Takahashi, D. 2005. Introduction to the HPC Challenge Benchmark Suite, Technical Report, ICL, University of Tennessee at Knoxville.

[13] Netlib. HPL Tuning http://www.netlib.org/benchmark/hpl/tuning.html\#tips

[14] Dunlop, D., Varrette, S. and Bouvry, P. 2008. On the use of a genetic algorithm in high performance computer benchmark tuning, Proceedings of the International Symposium on Performance Evaluation of Computer and Telecommunication Systems, SPECTS 2008, Art. No.:4667550, 105-113.

[15] HPL Frequently Asked Questions. [Online]. Available: http://www.netlib.org/benchmark/hpl/faqs.html

[16] Sindi, M. 2009. HowTo - High Performance Linpack (HPL), Technical Report, Center for Research Computing, University of Notre Dame.

[17] Petitet, A., Whaley, R. C., Dongarra, J., and Cleary, A. HPL - a portable implementation of the highperformance linpack benchmark for distributed-memory computers. http://www.netlib.org/benchmark/hpl/

[18] Cox J. Simon, Cox T. James, Boardman P. Richard, Johnston J. Steven, Scott Mark, and Neil S. O'Brien. Iridis-pi: a low-cost, compact demonstration cluster. Cluster Computing 17, no. 2 (June 22, 2013): 349-58 doi: 10.1007/s10586-013-0282-7. 